\title{
WITNESSING THE GRADUAL SLOWDOWN OF POWERFUL EXTRAGALACTIC JETS: THE X-RAY-OPTICAL-RADIO CONNECTION
}

\author{
Markos Georganopoulos ${ }^{i}$ and Demosthenes Kazanas \\ Laboratory for High Energy Astrophysics, NASA Goddard Space Flight Center, Code 661, Greenbelt, MD 20771; \\ markos@milkyway.gsfc.nasa.gov, demos.kazanas-1@ nasa.gov \\ Received 2004 January 14; accepted 2004 February 19; published 2004 March XX
}

\begin{abstract}
A puzzling feature of the Chandra-detected quasar jets is that their $\mathrm{X}$-ray emission decreases faster along the jet than their radio emission, resulting (in an outward-increasing radio-to-X-ray ratio. In some sources this behavior is so extreme that the radio emission peak is located clearly downstream of that of the X-rays. This is a rather unanticipated behavior given that the inverse Compton nature of the $\mathrm{X}$-rays and the synchrotron radio emission are attributed to roughly the same electrons of the jet's nonthermal electron distribution. In this Letter we show that this morphological behavior can result from the gradual deceleration of a relativistic flow and that the offsets in peak emission at different wavelengths carry the imprint of this deceleration. This notion is consistent with another recent finding, namely, that the jets feeding the terminal hot spots of powerful radio galaxies and quasars are still relativistic with Lorentz factors $\Gamma \sim 2-3$. The picture of the kinematics of powerful jets emerging from these considerations is that they remain relativistic as they gradually decelerate from kiloparsec scales to the hot spots, where, in a final collision with the intergalactic medium, they slow down rapidly to the subrelativistic velocities of the hot spot advance speed.
\end{abstract}

Subject headings: galaxies: active - quasars: general — radiation mechanisms: nonthermal — X-rays: galaxies

\section{INTRODUCTION}

The superior angular resolution and sensitivity of Chandra has led to the discovery of $\mathrm{X}$-ray emission from a number of quasar jets. Schwartz et al. (2000) were the first to note that the X-ray emission from the knots of the jet of the superluminal quasar PKS 0637-752 (Chartas et al. 2000), at a projected distance $\sim 100 \mathrm{kpc}$ from the quasar core, is part of a spectral component that is separate from its synchrotron radio-optical emission and is too bright to be explained by synchrotron selfCompton emission from electrons in energy equipartition with the jet magnetic field. Note, however, that in the innermost knot of some sources-e.g., 3C 273 (Marshall et al. 2001) and PKS 1136- 165 (Sambruna et al. 2002) - a synchrotron X-ray contribution is possible. These properties are apparently common to other quasars jets, as indicated by the mounting observational evidence (Sambruna et al. 2001; Marshall et al. 2001; Jester et al. 2002; Sambruna et al. 2002; Siemiginowska et al. 2002; Jorstad, Marscher, \& McHardy 2003; Siemiginowska et al. 2003; Yuan et al. 2003; Cheung 2004; Sambruna et al. 2004).

To account for the level of the observed X-ray emission, Tavecchio et al. (2000) and Celotti, Ghisellini, \& Chiaberge (2001) proposed that this is due to external Compton (EC) scattering of the cosmic microwave background (CMB) photons off relativistic electrons in the jet, provided that the jet flow is sufficiently relativistic $(T \sim 10)$ to boost the CMB energy density in the flow frame (by $\Gamma^{2}$ ) at the level needed to reproduce the observed $\mathrm{X}$-ray flux.

In all these sources the radio-to-X-ray ratio increases downstream along the jet, an unexpected behavior, given that the cooling length of the EC X-ray-emitting electrons ( $\gamma$ of about a few hundred) is longer than that of the radio-emitting ones the size of the jet, which would lead to a constant X-ray brightness as far out as the hot spots, contrary to observations. More surprisingly, in some jets (e.g., 3C 273 in Sambruna et al. 2001

\footnotetext{
'NAS/NRC Research Associate.
}

and Marshall et al. 2001; PKS 1136-135 and 1354+195 in Sambruna et al. 2002; PKS 1127-145 in Siemiginowska et al. 2002; and 0827+243 in Jorstad et al. 2003) the X-ray and radio maps are anticorrelated, with the $\mathrm{X}$-ray emission peaking closer to the core, gradually decreasing outward, and the radio emission increasing outward to peak practically at the very end of the radio jet.

To explain the reduction of the X-ray flux along the jet, Tavecchio, Ghisellini, \& Celotti (2003) suggested that the Xray emission originates in a collection of microknots undergoing adiabatic expansion sufficient to produce the desirable electron cooling. However, this would also suppress the radio emission, leading to practically indistinguishable radio and $\mathrm{X}$ ray morphologies, contrary to their observed spatial anticorrelation. An elegant suggestion by Dermer \& Atoyan (2002) that the $\mathrm{X}$-rays are synchrotron emission from electrons cooling in the Klein-Nishina regime naturally produces shorter sizes in $\mathrm{X}$-rays than in radio. However, it also produces larger optical than X-ray sizes, contrary to observations, and it seems therefore not to be applicable in this particular context.

Our view is that although $\mathrm{X}$-ray-producing electrons are present throughout the jet, the $\mathrm{X}$-ray brightness decreases because the CMB photon energy density in the flow frame decreases along the jet as a result of a decelerating relativistic jet flow. The decrease in the flow Lorentz factor leads to a decrease in the comoving $\mathrm{CMB}$ photon energy density and hence to a decrease in the $\mathrm{X}$-ray brightness along the jet. At the same time, the flow deceleration leads to a compression of the magnetic field; this results in an enhanced radio emission with distance, which thus gets displaced downstream of the EC Xray emission.

Based on the radio, optical, and X-ray jet maps, we argue in this Letter that powerful extragalactic jets are relativistic and gradually decelerating. In $\S 2$ we formulate the synchrotron and $\mathrm{EC}$ emission process from a decelerating jet flow and present our results. In $\S 3$ we discuss our findings and touch upon some open issues. 


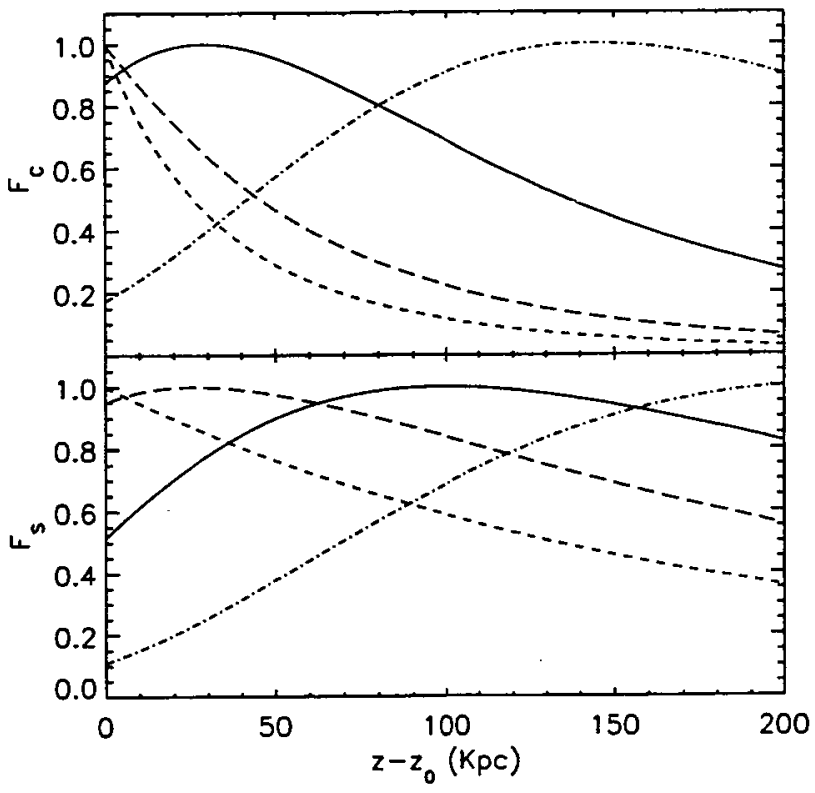

Fig. 1.-EC (upper panel) and synchrotron (lower panel) flux normalized to its maximum value for a range of observing angles along a jet that decelerates from $\Gamma_{0}=6$ to $\Gamma=2$ with $\epsilon=1$ from $z_{0}=100 \mathrm{kpc}$ to $z_{\max }=300 \mathrm{kpc}$, while its radius doubles. The observing angles are $\theta=0^{\circ}$ (short-dashed line), $\theta=1 / 2 \Gamma_{0} \approx 4.8$ (long-dashed line), $\theta=1 / \Gamma_{0} \approx 9.6$ (solid line), and $\theta=$ $2 / \Gamma_{0} \approx 19.5$ (dot-dashed line)

\section{THE MODEL}

We parameterize the jet flow assuming that its radius $r$ scales as $r(z)=r_{0} f(z)$, where $f(z)$ is a function with $f\left(z_{0}\right)=1$ and $r_{0}$ is the jet radius at a fiducial point $z_{0}$ along the jet. Similarly, we assume that the flow decelerates as $\Gamma(z)=\Gamma_{0} g(z)$, where $g(z)$ is a function with $g\left(z_{0}\right)=1$ and $\Gamma_{0}$ is the bulk Lorentz factor at $z_{0}$. The evolution of the electron energy distribution (EED) along the jet is determined by the combination of adiabatic changes (losses for expansion and gains for compression), radiative losses, and particle acceleration. If the radiative losses of the electrons responsible for the $\mathrm{X}$-ray and radio emission are negligible (as may be the case for the ( $\gamma$ of about a few hundred) EC X-ray-emitting electrons, or if we assume that some localized (e.g., shocks; Kirk et al. 2000) or spatially distributed (e.g., turbulence; Manolakou, Anastasiadis, \& Vlahos 1999) particle acceleration mechanism offsets the radiative losses, then the evolution of the EED can be treated as adiabatic. In this case, assuming that $\Gamma \gg 1$, the elementary volume $d V$ scales as $d V=d V_{0} f^{2} g$, allowing for expansion or compression, while the adiabatic electron energy change rate with $z$ is obtained from $\gamma^{\prime}=-\gamma\left(2 f^{\prime} / f+g^{\prime} / g\right) / 3$, where the prime denotes differentiation with respect to $z$. The solution of the above equation is $\gamma(z)=\gamma_{0} f^{-2 / 3} g^{-1 / 3}$. Assuming particle conservation, $n(\gamma, z) d V d \gamma=n\left(\gamma_{0}, z_{0}\right) d V_{0} d \gamma_{0}$, the comoving EED $n(\gamma, z)$ can be written as

$$
n(\gamma, z)=k \gamma^{-s} f^{-2(s+2) / 3} g^{-(s+2) / 3},
$$

where $n\left(\gamma, z_{0}\right)=k \gamma^{-s}$ is the EED at $z_{0}$. Following Georganopoulos, Kirk, \& Mastichiadis (2001), the EED in the local rest frame is $n(\gamma, z) \Delta^{2+s}$, where $\Delta(z)$ is the familiar Doppler factor $\Delta(z)=1 /\{\Gamma(z)[1-\beta(z) \cos \theta]\}$ and $\theta$ is the observing angle. Using this and taking into account the cosmological corrections, the EC flux per $d z$ in the $\delta$-function approximation (Coppi \& Blandford 1990; an electron of Lorentz factor $\gamma$ upscatters seed photons of energy $\epsilon_{0}$ only to an energy $4 \epsilon_{0} \gamma^{2 / 3)}$ is

$$
\frac{d F_{c}}{d \epsilon d z}=\frac{k \sigma_{7} c U(1+Z)^{2}}{2 d^{2} \epsilon_{0}} f^{-2(s-1) / 3} g^{-(s+2) / 3} \Delta^{2+s}\left(\frac{3 \epsilon}{4 \epsilon_{0}}\right)^{-(s-1) / 2},
$$

where $Z$ and $d$ are, respectively the redshift and proper distance of the source, $\epsilon_{0}$ and $\epsilon$ are, respectively, the energy of the CMB seed photons and the observed photons in units of $m_{e} c^{2}$ at $Z=0$, and $U$ is the $\mathrm{CMB}$ energy density at $Z=0$. For $\theta=0, \Delta(z)=2 \Gamma(z)$, and the $z$-dependence of the flux scales proportional to $f^{-2(s-1) / 3} g^{(4+2 s) / 3}$.

Assuming flux conservation, the magnetic field $B(z)$ can be written as $B(z)=B_{0} / f g$. The synchrotron flux per $d z$ in the $\delta$ function approximation (an electron of Lorentz factor $\gamma$ produces synchrotron photons only at the critical synchrotron en$\left.\operatorname{ergy} B \gamma^{2} / B_{\mathrm{cr}}\right)$ is then

$$
\begin{aligned}
\frac{d F_{s}}{d \epsilon d z}= & \frac{k \sigma_{\tau} c B_{c r} B_{0}^{(s+1) / 2}}{12 \pi d^{2}(1+Z)^{(s+1) / 2}} \\
& \times f^{-(7 s-1) / 6} g^{-(5 s+7) / 6} \Delta^{(s+3) / 2}\left(\epsilon B_{\mathrm{cr}}\right)^{-(s-1) / 2},
\end{aligned}
$$

where $B_{\mathrm{cr}}=m_{e}^{2} c^{3} / e \hbar=4.414 \times 10^{13} \mathrm{G}$ is the critical magnetic field. Note that while the synchrotron flux decreases with increasing redshift [the quantity $d^{2}(1+Z)^{(s+1) / 2}$ increases], the EC flux $\left[\propto(1+Z)^{2} / d^{2}\right]$, after an initial decrease up to $Z \approx$ 1.9 , remains practically constant for higher $Z$, as was first noted by Schwartz (2002).

For $\theta=0$ the $z$-dependence of the flux scales proportional to $f^{-(7 s-1) / 6} g^{-(s-1) / 3}$. The condition under which the synchroton flux increases along the jet at $\theta=0$ is that deceleration dominates over the jet opening, leading to $-g^{\prime} / g<(7 s-1) \times$ $f^{\prime} / 2(s-1) f$. Taking the ratio of equation (3) to equation (2), the condition under which $L_{s} / L_{\mathrm{EC}}$ (or, equivalently, the radioto-X-ray spectral index $\alpha_{\mathrm{rx}}$ ) increases with $z$ at $\theta=0$ is $-g^{\prime} / g<f^{\prime} / 2 f$, and for a given jet opening profile $f$, a milder deceleration $g$ is needed to produce an increase in $\alpha_{\mathrm{rx}}$ than to separate the $\mathrm{X}$-ray and radio peaks. This is in agreement with observations that show that the decline of the radio-to-X-ray flux is a more widely observed trend that the X-ray-radio peak displacement.

For the rest of this work, we consider conical jets with $f=(1+a z) /\left(1+a z_{0}\right)$, where $a=0$ corresponds to a cylindrical flow, and deceleration profiles $g=\left(z / z_{0}\right)^{-\epsilon}$, where $\epsilon \geq$ 0 . The behavior of the EC and synchrotron flux profiles also depends on the observing angle. To demonstrate this, we plot in the upper (lower) panel of Figure 1 the EC (synchrotron) flux along a decelerating jet, for a range of angles. It can be seen that while for small angles the EC flux peaks at the base of the jet, at larger angles the peak shifts downstream as a result of the fact that the emission from the faster base of the jet is beamed out of the line of sight [for a given $\theta, \Delta(z)$ peaks at $\Gamma(z) \approx 1 / \sin \theta]$. The behavior of the synchrotron flux is also interesting. At angles $\theta \neq 0$ the peak of the synchrotron emission shifts downstream, and for a given angle it is farther downstream than the EC peak, in agreement with observations.

It is interesting to see how much the above considerations change when radiative losses are important. The simplest case is that of a perfectly collimated (i.e., $\alpha=0$ ) cylindrical jet. This is also the case that requires the mildest deceleration for producing a given $\alpha_{\mathrm{rx}}$ increase or X-ray-radio separation. As- 


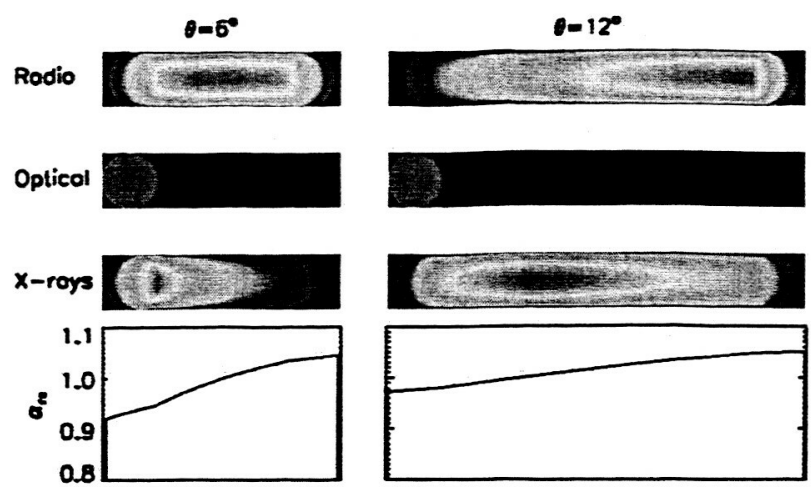

Fig. 2.-Radio (top), optical (middle), X-ray (bottom) maps and the radioto-X-ray spectral index $\alpha_{\mathrm{fx}}$ (below the X-ray map) for a decelerating flow observed under $\theta=6^{\circ}$ (left) and $\theta=12^{\circ}$ (right). The cylindrical flow, assumed to be at $Z=0.3$, decelerates from $\Gamma_{0}=6$ to $\Gamma=2$ with $\epsilon=1$ and $B_{0}=$ $3 \times 10^{-6}$ as it propagates a distance of $200 \mathrm{kpc}$. The EED is a power law of index $s=2$ and $\gamma_{\max }=10^{6}$ at the base of the flow. This calculation includes radiative losses. Each map is normalized, with red indicating the maximum luminosity per unit area. The projected jet lengths are $\approx 21 \mathrm{kpc}$ for $\theta=6^{\circ}$ and $\approx 42 \mathrm{kpc}$ for $\theta=12^{\circ}$.

suming an EED slope $s=2$, the EED remains a power law of the same siope following equation (1), with a maximum energy electron Lorentz factor

$$
\gamma_{\max }(z)=\frac{\gamma_{\max \left(z_{0}\right)} z_{\star}^{\epsilon / 3}}{1+\gamma_{\max \left(z_{0}\right)}\left[C_{1}\left(z_{\star}^{(10 \epsilon+3) / 3}-1\right)+C_{2}\left(z_{\star}^{(3-2 \epsilon) / 3}-1\right)\right]},
$$

where $\gamma_{\max \left(z_{0}\right)}$ is the upper cutoff of the EED at $z_{0}, z_{\star}=z / z_{0}$. $C_{1}=3 z_{0} A B_{0}^{2} / 8 \pi(10 \epsilon+3), C_{2}=4 z_{0} A U(1+Z)^{4} \Gamma_{0}^{2} /(3-2 \epsilon)$, and $A=4 \sigma_{\tau} / 3 m_{e} c^{2} \Gamma_{0}$. The maximum EC energy $\epsilon_{\mathrm{EC}, \max }$ as a function of $z$ is then $\epsilon_{E C \text {, max }}=\epsilon_{0} \Delta^{2}(z) \gamma_{\text {max }}^{2}(z)$, while the maximum synchrotron energy is $\epsilon_{s, \max }=B_{0} z_{\star}^{t} \Delta(z) \gamma_{\max }^{2}(z) / B_{\mathrm{cr}}$. For a given observing energy and observing angle, one can find the radiating part of the jet and perform the optically thin radiative transfer by integrating equations (2) and (3) along each line of sight for a two-dimensional array of lines of sight that covers the source as projected on the observer's plane of the sky.

The results of such calculations are shown in Figure 2, where it can be seen that the optical emission is confined at the base of the flow because of the strong radiative losses of the highenergy electrons, practically marking the site of strong particle acceleration. The morphology of the radio emission is angledependent: at $\theta=6^{\circ}$ it covers the entire extent of the jet, while at $\theta=12^{\circ}$ the emission of its inner part is dimmed because most of the radiation is beamed outside our line of sight. The $\mathrm{X}$-ray emission at both angles peaks close to the base of the flow because of the decrease of the comoving CMB photon energy density away from the flow base. Regarding the relative radio$\mathrm{X}$-ray morphology, while at $\theta=12^{\circ}$ the offset between the two images is very large, at $\theta=6^{\circ}$ the two images overlap, although the radio image clearly extends farther downstream. Also, as the radio-X-ray spectral index plots show, in both cases $\alpha_{\mathrm{rx}}$ increases downstream in agreement with observations.

We now compare this picture with the one we would get from the same system if radiative losses were balanced by distributed reacceleration (Fig. 3). The X-ray images remain practically the same because the radiative losses for the EC Xray-emitting electrons in this $Z=0.3, \Gamma_{0}=6$ jet are negli-

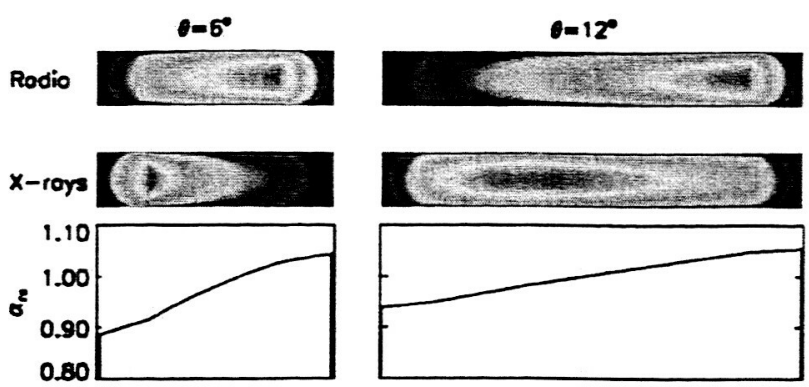

Fig. 3.-Same as in Fig. 2, but with radiative losses balanced by distributed reacceleration and without the optical map, which in this case would be identical to the radio map.

gible. The radio image, however, changes significantly, particularly so for the $\theta=6^{\circ}$ orientation. Now that the radio-emitting electrons retain their energy, the outer part of the jet is much brighter than the inner part, resulting in a clear separation between the radio and $\mathrm{X}$-ray images.

\section{DISCUSSION AND CONCLUSIONS}

We have proposed that the increase of the radio-to-X-ray fiux ratio along the length of the jets of powerful quasars and the occasional offset of the jet images in these wavelengths are naturally accounted for in terms of relativistic flows that decelerate over the entire length of the jet. Despite this deceleration, the jets remain relativistic ( $\Gamma$ of about a few) to their terminal hot spots (Georganopoulos \& Kazanas 2003), within which they eventually attain subrelativistic speeds. Our proposal provides, for the first time, a means for deducing the jet kinematics through simple models of their multiwavelength images; these can then be checked for consistency when coupled with the detailed hot spot emission, which, as shown in earlier work (Georganopoulos \& Kazanas 2003), depends on the value of $\Gamma$ at this location. We note here that a change of the Doppler factor $\Delta$ can also result from a jet curving away from (or into) the observer's line of sight without actually decelerating. In this case, due to the stronger dependence of EClon $\Delta$ (Dermer 1995; Georganopoulos et al. 2001), it is also possible to obtain increasing (or decreasing, which is not observed) radio-to-X-ray ratios; however, because the comoving $\mathrm{CMB}$ photon density and magnetic fields remain constant in this case, such an arrangement cannot produce the observed displacements between the X-ray and radio peak emissions.

The scenario we propose here finds further support from modeling jets with several sequential individual knots: Sambruna et al. (2001) noted the need for a gradual decrease of the Doppler factor and/or an increase of the magnetic field in order to reproduce the emission from the knots along the jet of 3C 273 with simple one-zone models. These knot-to-knot variations can be naturally incorporated within the context of a decelerating collimated flow, as we propose.

These same maps also may or may not indicate the need for distributed particle reacceleration along these jets, when the EC loss length scale of their radio-emitting electrons $[\propto(1+$ $Z)^{-4} \Gamma^{-2}$ ] is shorter than the observed length of the radio jet. This appears to be the case with sources like PKS 1127-145 at $Z=1.187$ (Siemiginowska et al. 2002) and possibly GB $1508+5714$ at $Z=4.3$ (Yuan et al. 2003; Siemiginowska et al. 2003; Cheung 2004), which show their peak radio emission displaced from that of the X-rays: in the absence of reacceleration, EC losses of the radio-emitting electrons would pro- 
duce radio jets shorter than X-ray jets. Much about the reacceleration process of these jets can be inferred from their morphology at different frequencies. For example, the knotty optical jet morphology shows that reacceleration is not strong enough to offset the EC-dominated losses of the optically emitting synchrotron electrons $\left(\gamma \sim 10^{6}-10^{7}\right)$.

The radiative efficiency of these jets is less than a few percent (e.g., Tavecchio et al. 2000), and the energy lost in deceleration must be either used to heat up the matter in the jet or transferred to material that is entrained by the jet. While the first option would result in an expansion of the jet, contrary to what is seen, entrainment from an external medium would load the jet with baryonic mass while decelerating it. Entrainment would produce velocity gradients across the jet, and in this sense a faster spine and a slower sheath are to be expected. However, the observed X-ray and radio jet morphologies suggest that the dominant effect must be a deceleration along the jet. A fast spine that does not decelerate substantially and carries most of the jet power would produce a constant X-ray EC and radio synchrotron flux along the jet, in disagreement with observations. A consequence of entrainment would be that even if the jet did not start as a baryonic one, entrainment would gradually enrich it with baryons and eventually a fraction $\Delta \Gamma / \Gamma$ of its power, where $\Delta \Gamma$ is the decrease of the Lorentz factor, would be carried by the entrained baryonic matter. This in turn would increase the radio lobe equipartition energy content estimates derived under the assumption of a leptonic composition. Our findings point to a picture in which powerful relativistic jets decelerate, depositing most of their power in their surroundings in the form of kinetic energy, their observed radiation being only the tip of the iceberg.

We want to thank the referee for his/her suggestions. G. M. thanks Rita Sambruna and Jessica Gambill for illuminating discussions on recent jet observations.

\section{REFERENCES}

Celotti, A., Ghisellini, G., \& Chiaberge, M. 2001, MNRAS, 321, L1

Chartas, G., et al. 2000, ApJ, 542, 655

Cheung, C. C. 2004, ApJ, 600, L23

Coppi, P. S., \& Blandford, R. D. 1990, MNRAS, 245, 453

Dermer, C. D. 1995 , ApJ, 446, L63

Dermer, C. D., \& Atoyan, A. M. 2002, ApJ, 568, L81

Georganopoulos, M., \& Kazanas, D. 2003, ApJ, 589, L5

Georganopoulos, M., Kirk, J. G., \& Mastichiadis, A. 2001, ApJ, 561, 111

Jester, S., Röser, H.-J., Meisenheimer, K., \& Perley, R. 2002, A\&A, 385, L27

Jorstad, S. G., Marscher, A. P., \& McHardy, I. 2003, AAS Meeting, 202, 43.01

Kirk, J. G., Guthmann, A. W., Gallant, Y. A., \& Achterberg, A. 2000, ApJ, 542,235

Manolakou, K., Anastasiadis, A., \& Vlahos, L. 1999, A\&A, 345, 653

Marshall, H. L., et al. 2001, ApJ, 549, L167

Sambruna, R. M., Gambill, J. K., Maraschi, L., Tavecchio, F., Cerutti, R., Cheung, C. C., Urry, C. M., \& Chartas, G. 2004, ApJ, in press (astro-ph/ 0401475)
Sambruna, R. M., Maraschi, L., Tavecchio, F., Urry, C. M., Cheung, C. C., Chartas, G., Scarpa, R., \& Gambill, J. K. 2002, ApJ, 571, 206

Sambruna, R. M., Urry, C. M., Tavecchio, F., Maraschi, L., Scarpa, R., Chartas, G., \& Muxlow, T. 2001, ApJ, 549, L161

Siemiginowska, A., Bechtold, J., Alderoft, T. L., Elvis, M., Harris, D. E., \& Dobrzycki, A. 2002, ApJ, 570, 543

Siemiginowska, A., Smith R. K., Aldcroft T. L., Schwartz, D. A., Paerels, F., \& Petric, A. O. 2003, ApJ, 598, L15

Schwartz, D. E. 2002, ApJ, 569, L23

Schwartz, D. E., et al. 2000, ApJ, 540, L69

Tavecchio, F., Ghisellini, G., \& Celotti, A. 2003, A\&A, 403, 83

Tavecchio, F., Maraschi, L., Sambruna, R., \& Urry, C. M. 2000, ApJ, 544, L23

Yuan, W., Fabian, A. C., Celotti, A., \& Jonker, P. G. 2003, MNRAS, 346, L7 